STUPP-07-193

October, 2007

\title{
Chiral Symmetry Breaking in Brane Models
}

\author{
Norio Horigome, , $^{*}$ Madoka Nishimura ${ }^{\mathrm{b}}$ and Yoshiaki Tania ${ }^{\oplus \dagger}$ \\ ${ }^{a}$ Division of Material Science \\ Graduate School of Science and Engineering \\ Saitama University, Saitama 338-8570, Japan \\ ${ }^{\mathrm{b}}$ Department of Community Service and Science \\ Tohoku University of Community Service and Science \\ Iimoriyama 3-5-1, Sakata 998-8580, Japan
}

\begin{abstract}
We discuss the chiral symmetry breaking in general intersecting $\mathrm{D} q / \mathrm{D} p$ brane models consisting of $N_{c} \mathrm{D} q$-branes and a single $\mathrm{D} p$-brane with an $s$-dimensional intersection. There exists a QCD-like theory localized at the intersection and the $\mathrm{D} q / \mathrm{D} p$ model gives a holographic description of it. The rotational symmetry of directions transverse to both of the $\mathrm{D} q$ and $\mathrm{D} p$-branes can be identified with a chiral symmetry, which is non-Abelian for certain cases. The asymptotic distance between the $\mathrm{D} q$-branes and the $\mathrm{D} p$-brane corresponds to a quark mass. By studying the probe $\mathrm{D} p$-brane dynamics in a $\mathrm{D} q$-brane background in the near horizon and large $N_{c}$ limit we find that the chiral symmetry is spontaneously broken and there appear (pseudo-)Nambu-Goldstone bosons. We also discuss the models at finite temperature.
\end{abstract}

\footnotetext{
* e-mail: horigome@krishna.th.phy.saitama-u.ac.jp

$\dagger$ e-mail: tanii@phy.saitama-u.ac.jp
} 


\section{Introduction}

The AdS/CFT correspondence [1, 2, 3] (see [4] for a review) provides a new nonperturbative approach to strongly coupled gauge theories. This duality relates a string theory in $(d+1)$-dimensional anti de Sitter spacetime (times a compact space) to a $d$-dimensional conformal field theory. The AdS/CFT correspondence can be extended to the string/gauge duality, which is a generalization to non-conformal and non-supersymmetric theories. The string/gauge duality also provides us with a useful tool for the analysis of low energy behaviors of QCD such as the confinement and the spontaneous chiral symmetry breaking. This approach is often called the holographic QCD [5, 6, 7, 8, 9, 10, 11, 12, 13, 14] (and references therein).

One of the most interesting phenomena of the low energy QCD is the spontaneous breaking of the chiral symmetry. In the holographic approach the chiral symmetry can be realized in two different ways. In this approach one introduces $N_{c}$ color $\mathrm{D} q$ branes and $N_{f}$ flavor D $p$-branes. The $\mathrm{U}\left(N_{c}\right)$ gauge field on the $\mathrm{D} q$-branes represents a gluon field of a QCD-like theory. Open strings connecting the D $q$-branes and the $\mathrm{D} p$-branes represent quarks in the fundamental representation of $\mathrm{U}\left(N_{c}\right)$. When these brane configurations have directions transverse to both of the $\mathrm{D} q$ and $\mathrm{D} p$-branes, a rotational symmetry in these directions can be understood as a chiral symmetry of the dual gauge theories in certain cases [7, 8, 9, 10, 11, 12]. One can separate color branes and flavor branes in such directions. This is a holographic description of the spontaneous chiral symmetry breaking. The asymptotic distance between these branes is identified with a quark mass. So one can study the chiral symmetry breaking with a non-vanishing quark mass. So far only the Abelian chiral symmetry $\mathrm{U}(1)_{V} \times \mathrm{U}(1)_{A}$ is considered in this approach.

Alternatively, the chiral symmetry can be realized as a gauge symmetry on the flavor branes. When $\mathrm{D} p-\overline{\mathrm{D} p}$-brane pairs are used as flavor branes, one can obtain a non-Abelian $\mathrm{U}\left(N_{f}\right)_{L} \times \mathrm{U}\left(N_{f}\right)_{R}$ chiral symmetry [13, 14, 15, 16, 17, 18, 19, 20]. These configurations of physical interest often do not have directions transverse to both of the color and flavor branes. Therefore, it is not obvious how to introduce a quark mass in these models. For work toward an introduction of a quark mass in this type of models and on related issues see refs. [21, 22, 23, 24, 25]. In both of these two approaches, the spontaneous breaking of the chiral symmetry is closely related to the configurations of the probe branes in the background geometry.

The chiral symmetry breaking was also discussed at finite temperature [7, 8, 26, 
[27, 28, 29, 30, 31] and at finite chemical potential [32, 33, 34, 35, 36, 37]. The temperature $T$ is related to a period $\delta t_{E}$ of the $\mathrm{S}^{1}$ compactified Euclidean time coordinate as $T=1 / \delta t_{E}$. The chemical potential $\mu$ is introduced as a non-vanishing asymptotic value of the time component of the gauge field on the probe brane $A_{0} \sim \mu$. One can study a chiral phase transition and obtain a phase diagram of the QCD-like theories.

The purpose of the present paper is to study the chiral symmetry breaking in general intersecting $\mathrm{D} q / \mathrm{D} p$ brane systems consisting of $N_{c}$ color $\mathrm{D} q$-branes and a single probe $\mathrm{D} p$-brane with an $s$-dimensional intersection. They are holographic duals of QCD-like theories in $(s+1)$-dimensional spacetime: $\mathrm{QCD}_{s+1}$. As in refs. [7, 8] these models can have directions transverse to both of the $\mathrm{D} q$ and $\mathrm{D} p$-branes. A rotational symmetry of these directions can be interpreted as a chiral symmetry in certain cases. This symmetry can be non-Abelian in contrast to the models in refs. [7, 8. We can separate the $\mathrm{D} q$-branes and the $\mathrm{D} p$-brane in these transverse directions and break the rotational symmetry. In the holographic description this deformation makes quarks on the intersection massive and leads to an explicit chiral symmetry breaking. In the near horizon limit and the large $N_{c}$ limit we can treat the $\mathrm{D} q$-branes as a background geometry and the $\mathrm{D} p$-brane as a probe which does not affect this background. We discuss the chiral symmetry breaking by analyzing the $\mathrm{D} p$-brane dynamics in the $\mathrm{D} q$-brane background geometry.

The organization of this paper is as follows. In sect. 2 we study the low energy spectrum at an $s$-dimensional intersection of the $\mathrm{D} q / \mathrm{D} p$ brane system. There are a number of brane systems corresponding to QCD-like theories at the intersection. We can classify these theories by a pair of numbers $(q+p, s)$. In particular, the classes of configurations belonging to $(6,1),(8,2)$ and $(10,3)$ correspond to $\mathrm{QCD}_{2}$, $\mathrm{QCD}_{3}$ and $\mathrm{QCD}_{4}$, respectively. For particular $(q+p, s)$ the rotational symmetry of the transverse directions can be understood as a chiral symmetry in the QCD-like theories. This chiral symmetry is non-Abelian $\mathrm{SU}(2)_{L} \times \mathrm{SU}(2)_{R}$ for $\mathrm{QCD}_{2}$.

In sect. 3 we study the chiral symmetry breaking in the QCD-like theories by using a supergravity analysis. The near horizon limit and the large $N_{c}$ limit $N_{c} \gg 1$ allow us to study the probe $\mathrm{D} p$-brane dynamics in the $\mathrm{D} q$-brane background. We find that a $\mathrm{D} p$-brane embedding breaks the rotational symmetry of the transverse directions. This corresponds to a chiral symmetry breaking in the QCD-like theories. The quark mass $m_{q}$ and the quark condensate $\langle\bar{\psi} \psi\rangle$ can be read from the asymptotic behavior of the $\mathrm{D} p$-brane embedding. There is a non-zero value of the quark 
condensate $\langle\bar{\psi} \psi\rangle$ even for the massless quark limit. This leads to a spontaneous chiral symmetry breaking in the QCD-like theories.

In sect. 4 we consider fluctuations of the $\mathrm{D} p$-brane around the vacuum embedding discussed in sect. 3. For $m_{q}=0$ there appear $(8-q-p+s)$ massless scalar bosons, which can be understood as the Nambu-Goldstone (NG) bosons associated with the spontaneous symmetry breaking. For a non-zero but small quark mass there appear pseudo-NG bosons. We show that these pseudo-NG bosons satisfy the GellMann-Oakes-Renner (GMOR) relation [38]. The effective action of the fluctuations is obtained at quartic order.

In sects. 3 and 4 we consider the case in which the dimension of the intersection $s$ is smaller than $q$. The case $s=q$ is treated in sect. 5. D $p$-brane embeddings in this case have different behaviors from those of $s<q$. They are similar to embeddings at finite temperature.

In sect. 6 we discuss the theories at finite temperature. We study the probe $\mathrm{D} p$ brane dynamics in the Euclidean $\mathrm{D} q$ background. The $\mathrm{D} p$-brane embedding breaks the rotational symmetry as in the zero temperature case. Then the chiral symmetry is also broken at finite temperature. We find that the quark condensate vanishes and the chiral symmetry is restored only in the high temperature limit. We conclude in sect. 7 .

\section{General setup}

We consider an intersecting brane system consisting of $N_{c}$ color $\mathrm{D} q$-branes and a single probe $\mathrm{D} p$-brane

\begin{tabular}{rcccccccccccc} 
& $x^{0}$ & $\cdots$ & $x^{s}$ & $x^{s+1}$ & $\cdots$ & $x^{q}$ & $x^{q+1}$ & $\cdots$ & $x^{q+p-s}$ & $x^{q+p-s+1}$ & $\cdots$ & $x^{9}$ \\
\hline$N_{c} \mathrm{D} q$ & $\circ$ & $\cdots$ & $\circ$ & $\circ$ & $\cdots$ & $\circ$ & - & $\cdots$ & - & - & $\cdots$ & - \\
$\mathrm{D} p$ & $\circ$ & $\cdots$ & $\circ$ & - & $\cdots$ & - & $\circ$ & $\cdots$ & $\circ$ & - & $\cdots$ & -
\end{tabular}

with $x^{q}$ being a coordinate of $\mathrm{S}^{1}$. It has an $s$-dimensional intersection in the directions $x^{1}, \cdots, x^{s}$. The configuration (2.1) is a T-dual of D $s^{\prime} / \mathrm{D} 9$ system with $s^{\prime}=9-(q+p-2 s) \geq s$. Following ref. [19] we call it a transverse intersection if $s^{\prime}=s(q+p-s=9)$ and a non-transverse intersection if $s^{\prime}>s(q+p-s<9)$. Non-transverse intersections have directions transverse to both of the $\mathrm{D} q$-branes and 
the $\mathrm{D} p$-brane, while transverse intersections do not. We first consider the case $s<q$. The case $s=q$ will be discussed in sect. 5 .

The configuration (2.1) has the following symmetries. The gauge symmetry of this system is $\mathrm{U}\left(N_{c}\right) \times \mathrm{U}(1)$. The $\mathrm{U}(1)$ gauge symmetry on the Dp-brane is regarded as a global symmetry (baryon number symmetry) of an $(s+1)$-dimensional field theory at the intersection. The ten-dimensional Lorentz symmetry $\operatorname{SO}(1,9)$ is broken to its subgroup by the configuration (2.1). Therefore the global symmetry preserved at the intersection contains

$$
\mathrm{SO}(1, s) \times \mathrm{SO}(9-q-p+s) \times \mathrm{U}(1)
$$

where $\mathrm{SO}(1, s)$ is the Lorentz symmetry at the intersection and $\mathrm{SO}(9-q-p+s)$ is the rotational symmetry in the directions $x^{q+p-s+1}, \cdots, x^{9}$.

The spectrum of the theory localized at the intersection is as follows. Massless fields generated by $q-q$ strings (open strings having both ends on the D $q$-branes) are a gauge field $A_{\mu}(\mu=0,1, \cdots, s)$, scalar fields $\Phi^{i}(i=s+1, \cdots, 9)$ and fermionic fields $S$. Imposing the periodic boundary condition for the bosonic fields and the anti-periodic one for the fermionic fields along the compact $x^{q}$ direction, the fermions become massive at zero mode and supersymmetry is explicitly broken at low energy. Then the scalars acquire mass at one-loop level. Thus only the gauge field $A_{\mu}$ is massless at low energy. This gives a pure $\mathrm{U}\left(N_{c}\right)$ gauge theory.

To study the lowest modes generated by $q-p$ strings (open strings connecting the $\mathrm{D} q$-branes and the $\mathrm{D} p$-brane), we note the zero-point energy in the $\mathrm{R}$ sector and the NS sector 39]

$$
a^{\mathrm{R}}=0, \quad a^{\mathrm{NS}}=\frac{\# \mathrm{ND}-4}{8},
$$

where \#ND $=q+p-2 s=9-s^{\prime}$ is the number of spatial coordinates of open strings which have the Neumann boundary condition for one end and the Dirichlet one for the other end. The lowest modes generated by $q-p$ strings in the NS sector are massive for \#ND $>4(\# \mathrm{ND}=6,8)$, massless for $\# \mathrm{ND}=4$ and tachyonic for $\# \mathrm{ND}<4(\# \mathrm{ND}=0,2)$. We do not consider the tachyonic case $\# \mathrm{ND}<4$. When $\# \mathrm{ND} \geq 4$, the lowest modes from the NS sector are massive (by loop effects for $\# \mathrm{ND}=4$ ) and are decoupled at low energy. There are only massless fermions from the $\mathrm{R}$ sector. They belong to representations of the Clifford algebra for the NN and DD directions. These fermions belong to the fundamental representation of $\mathrm{U}\left(N_{c}\right)$ and are called "quarks". Therefore the effective theory on the intersection at 


\begin{tabular}{|c|c|cccccccccc||c||c|}
\hline & & 0 & 1 & 2 & 3 & 4 & 5 & 6 & 7 & 8 & 9 & $a^{\mathrm{NS}}$ & $(q+p, s)$ \\
\hline color & D2 & $\circ$ & $\circ$ & $\circ$ & - & - & - & - & - & - & - & & \\
\hline probe & D4 & $\circ$ & $\circ$ & - & $\circ$ & $\circ$ & $\circ$ & - & - & - & - & 0 & $(6,1)$ \\
& D6 & $\circ$ & $\circ$ & - & $\circ$ & $\circ$ & $\circ$ & $\circ$ & $\circ$ & - & - & $\frac{1}{4}$ & $(8,1)$ \\
\hline \hline color & D3 & $\circ$ & $\circ$ & $\circ$ & $\circ$ & - & - & - & - & - & - & & \\
\hline probe & D5 & $\circ$ & $\circ$ & $\circ$ & - & $\circ$ & $\circ$ & $\circ$ & - & - & - & 0 & $(8,2)$ \\
& D7 & $\circ$ & $\circ$ & $\circ$ & - & $\circ$ & $\circ$ & $\circ$ & $\circ$ & $\circ$ & - & $\frac{1}{4}$ & $(10,2)$ \\
\hline \hline color & D4 & $\circ$ & $\circ$ & $\circ$ & $\circ$ & $\circ$ & - & - & - & - & - & & \\
\hline probe & D6 & $\circ$ & $\circ$ & $\circ$ & $\circ$ & - & $\circ$ & $\circ$ & $\circ$ & - & - & 0 & $(10,3)$ \\
\hline
\end{tabular}

Table 1: A representative $\mathrm{D} q / \mathrm{D} p$ brane configuration for the class $(q+p, s)$ dual to $\mathrm{QCD}_{s+1}$.

low energy is an $(s+1)$-dimensional non-supersymmetric $\mathrm{U}\left(N_{c}\right)$ gauge theory with quarks in the fundamental representation. We call this theory " $\mathrm{QCD}_{s+1}$ " for the sake of convenience.

We are interested in non-transverse intersections satisfying $s^{\prime}>s$, which implies $s<9-$ \#ND. Possible cases are $s=1,2$ for \#ND $=6$ and $s=1,2,3,4$ for $\# \mathrm{ND}=4$. We further restrict ourselves to the cases $s=1,2,3$ since we are especially interested in $\mathrm{QCD}_{2}, \mathrm{QCD}_{3}$ and $\mathrm{QCD}_{4}$. There are a number of such $\mathrm{D} q / \mathrm{D} p$ configurations which are dual to $\mathrm{QCD}_{s+1}$. They can be classified by a pair of numbers $(q+p, s)=(\# \mathrm{ND}+2 s, s)$. Configurations belonging to the same class $(q+p, s)$ are equivalent through the T-duality in the ND directions. Therefore theories localized at the intersection for the same $(q+p, s)$ have the same low energy spectrum under the global symmetry (2.2). For example, in the $\# \mathrm{ND}=4$ and $s=1$ case, which gives $\mathrm{QCD}_{2}$, there are equivalent five configurations which belong to the class $(q+p, s)=(6,1)$. They are D1/D5, D2/D4, D3/D3, D4/D2 and D5/D1 configurations with a one-dimensional intersection. We have shown a representative configuration for each class in Table 1,

Since these configurations are non-transverse intersections, there are directions transverse to both of the $\mathrm{D} q$-branes and the $\mathrm{D} p$-brane. In refs. [7, 8] the rotational symmetry $\mathrm{SO}(9-q-p+s)$ of such directions is interpreted as a chiral symmetry in the dual gauge theory for certain sets of $(q+p, s)$. When the $\mathrm{D} q$-branes and the $\mathrm{D} p$-brane are separated along these directions, quarks on the intersection become massive and the chiral symmetry is explicitly broken. As we will see in sect. 3, only in the case $a^{\mathrm{NS}}=0(\# \mathrm{ND}=4)$ and $p-s-2>0$ an equation for probe 
brane embeddings derived from the DBI action has a solution for which the distance between the color branes and the probe brane asymptotically approaches a constant value. This distance is interpreted as a quark mass. Such configurations belong to the classes $(q+p, s)=(6,1),(8,2),(10,3)$, and are dual to $\mathrm{QCD}_{2}, \mathrm{QCD}_{3}$ and $\mathrm{QCD}_{4}$, respectively.

We can explicitly write down the symmetry (2.2) for the classes $(q+p, s)=(6,1)$, $(8,2),(10,3)$. In the class $(6,1)$, which has a one-dimensional intersection and is dual to $\mathrm{QCD}_{2}$, we can identify the $\mathrm{SO}(4)_{6789}$ rotational symmetry in the $x^{6}, x^{7}, x^{8}$, $x^{9}$ directions with an $\mathrm{SU}(2)_{L} \times \mathrm{SU}(2)_{R}$ chiral symmetry of quarks. Indeed, the GSO projection in the $R$ sector of open strings requires that the chiralities of $\mathrm{SO}(1,1)_{01}$ and $\mathrm{SO}(4)_{6789}$ are correlated. Left-handed (right-handed) quarks of $\mathrm{SO}(1,1)_{01}$ have the positive (negative) chirality of $\mathrm{SO}(4)_{6789}$ and transform as $(\mathbf{2}, \mathbf{1})((\mathbf{1}, \mathbf{2}))$ under $\mathrm{SU}(2)_{L} \times \mathrm{SU}(2)_{R}$. The gauge symmetry $\mathrm{U}(1)$ on the probe brane acts on quarks as a baryon number symmetry $\mathrm{U}(1)_{V}$. Therefore the global symmetry (apart from the Lorentz symmetry) of $\mathrm{QCD}_{2}$ at the intersection is

$$
\mathrm{SO}(4)_{6789} \times \mathrm{U}(1) \sim \mathrm{SU}(2)_{L} \times \mathrm{SU}(2)_{R} \times \mathrm{U}(1)_{V}
$$

Thus we can realize a non-Abelian chiral symmetry in a holographic model of this type, although spacetime is two-dimensional.

In the class $(8,2)$, which has a two-dimensional intersection and is dual to $\mathrm{QCD}_{3}$, we can identify the $\mathrm{SO}(3)_{789}$ rotational symmetry in the $x^{7}, x^{8}, x^{9}$ directions with an $\mathrm{SU}(2)$ symmetry of $\mathrm{QCD}_{3}$. Then the global symmetry of $\mathrm{QCD}_{3}$ at the intersection is

$$
\mathrm{SO}(3)_{789} \times \mathrm{U}(1) \sim \mathrm{SU}(2) \times \mathrm{U}(1) .
$$

Quarks transform as 2 under $\mathrm{SU}(2)$. Note that there is no chirality in $\mathrm{QCD}_{3}$ and therefore the symmetry (2.5) is not a chiral symmetry.

Finally, in the class $(10,3)$, which has a three-dimensional intersection and is dual to $\mathrm{QCD}_{4}$, we can identify the $\mathrm{SO}(2)_{89}$ rotational symmetry in the $x^{8}, x^{9}$ directions with an axial $\mathrm{U}(1)_{A}$ symmetry of $\mathrm{QCD}_{4}$ as discussed in refs. [7, 8]. The global symmetry of $\mathrm{QCD}_{4}$ at the intersection is

$$
\mathrm{SO}(2)_{89} \times \mathrm{U}(1) \sim \mathrm{U}(1)_{A} \times \mathrm{U}(1)_{V}
$$




\section{Chiral symmetry breaking from supergravity analysis}

The dynamics of a strongly coupled large $N_{c}$ gauge theory can be analyzed by supergravity. We study the chiral symmetry breaking in this section. The near horizon limit and the large $N_{c}$ limit $N_{c} \gg 1$ allow us to treat the $\mathrm{D} q$-branes as a background geometry and the $\mathrm{D} p$-brane as a probe which does not affect this background. We will find that the $\mathrm{D} p$-brane embedding breaks the $\mathrm{SO}(9-q-p+s)$ rotational symmetry in the directions transverse to both of the branes. This can be interpreted as the chiral symmetry breaking in $\mathrm{QCD}_{2}$ and $\mathrm{QCD}_{4}$. We will calculate the quark condensate and find a non-zero value even in the massless quark limit.

\subsection{D $q$-brane background}

The near horizon geometry of $\mathrm{S}^{1}$ compactified $N_{c} \mathrm{D} q$-branes is [40]

$$
\begin{gathered}
d s^{2}=\left(\frac{U}{R}\right)^{\frac{7-q}{2}}\left(-d t^{2}+\sum_{i=1}^{q-1}\left(d x^{i}\right)^{2}+f(U)\left(d x^{q}\right)^{2}\right)+\left(\frac{R}{U}\right)^{\frac{7-q}{2}}\left(\frac{d U^{2}}{f(U)}+U^{2} d \Omega_{8-q}^{2}\right) \\
f(U)=1-\left(\frac{U_{K K}}{U}\right)^{7-q}, \quad R^{7-q}=(4 \pi)^{\frac{5-q}{2}} \Gamma\left(\frac{7-q}{2}\right) g_{s} N_{c} \ell_{s}^{7-q}
\end{gathered}
$$

where $d \Omega_{8-q}^{2}, g_{s}$ and $\ell_{s}$ are the metric of a unit $S^{8-q}$, the string coupling and the string length, respectively. $x^{q}$ is a coordinate of $\mathrm{S}^{1}$ and its period is denoted as $\delta x^{q}=2 \pi / M_{K K} . M_{K K}$ is a compactification scale. To avoid a conical singularity at $U=U_{K K}$ in the $U-x^{q}$ plane the period must be related to a constant $U_{K K}$ as

$$
\delta x^{q}=\frac{4 \pi R^{\frac{7-q}{2}}}{(7-q) U_{K K}^{\frac{5-q}{2}}} .
$$

The dilaton field and the Ramond-Ramond (RR) flux are given by

$$
e^{\phi}=g_{s}\left(\frac{R}{U}\right)^{\frac{(7-q)(3-q)}{4}}, \quad F_{8-q}=\frac{N_{c}}{V_{8-q}} \epsilon_{8-q},
$$

where $\epsilon_{8-q}$ and $V_{8-q}$ are the volume form and the volume of a unit $\mathrm{S}^{8-q}$.

The relations between the parameters in the gauge theory and those in the string theory are

$$
g_{q+1}^{2}=(2 \pi)^{q-2} g_{s} \ell_{s}^{q-3}, \quad M_{K K}=\frac{7-q}{2(4 \pi)^{\frac{5-q}{4}} \Gamma\left(\frac{7-q}{2}\right)^{\frac{1}{2}}} \frac{U_{K K}^{\frac{5-q}{2}}}{\left(g_{s} N_{c}\right)^{\frac{1}{2}} \ell_{s}^{\frac{7-q}{2}}},
$$


where $g_{q+1}$ is the $(q+1)$-dimensional gauge coupling. The $(q+1)$-dimensional 't Hooft coupling is defined as

$$
\lambda_{q+1}=\frac{g_{q+1}^{2} N_{c}}{(2 \pi)^{q-2}} .
$$

Note that the supergravity description is valid for [40, 19]

$$
1 \ll \lambda_{q+1}\left(\frac{U_{K K}}{\ell_{s}^{2}}\right)^{q-3} \ll N_{c}^{\frac{4}{7-q}} .
$$

We introduce isotropic coordinates in the directions $\left(U, \Omega_{8-q}\right)$ to simplify the following analysis. Introducing a new radial coordinate $\rho$ defined by

$$
U=\left(\rho^{\frac{7-q}{2}}+\frac{U_{K K}^{7-q}}{4 \rho^{\frac{7-q}{2}}}\right)^{\frac{2}{7-q}}, \quad \rho^{2}=\sum_{\alpha=q+1}^{9}\left(x^{\alpha}\right)^{2}
$$

the metric for the transverse space $\left(U, \Omega_{8-q}\right)$ in eq. (3.1) can be written as

$$
\begin{aligned}
\left(\frac{R}{U}\right)^{\frac{7-q}{2}}\left(\frac{d U^{2}}{f(U)}+U^{2} d \Omega_{8-q}^{2}\right) & =K(\rho)\left(d \rho^{2}+\rho^{2} d \Omega_{8-q}^{2}\right) \\
& =K(\rho) \sum_{\alpha=q+1}^{9}\left(d x^{\alpha}\right)^{2}
\end{aligned}
$$

where

$$
K(\rho)=\frac{R^{\frac{7-q}{2}} U^{\frac{q-3}{2}}}{\rho^{2}} .
$$

We divide the coordinates $x^{q+1}, \cdots, x^{9}$ into two parts and introduce spherical coordinates $\left(\lambda, \Omega_{p-s-1}\right)$ for the $x^{q+1}, \cdots, x^{q+p-s}$ directions and $\left(r, \Omega_{8-q-p+s}\right)$ for the $x^{q+p-s+1}, \cdots, x^{9}$ directions. Then the $\mathrm{D} q$ background becomes

$$
\begin{aligned}
d s^{2}= & \left(\frac{U}{R}\right)^{\frac{7-q}{2}}\left(-d t^{2}+\sum_{i=1}^{q-1}\left(d x^{i}\right)^{2}+f(U)\left(d x^{q}\right)^{2}\right) \\
& +K(\rho)\left(d \lambda^{2}+\lambda^{2} d \Omega_{p-s-1}^{2}+d r^{2}+r^{2} d \Omega_{8-q-p+s}^{2}\right)
\end{aligned}
$$

where $\rho^{2}=\lambda^{2}+r^{2}$. We will wrap the probe $\mathrm{D} p$-brane around $\mathrm{S}^{p-s-1}$ in the next subsection. 


\section{2 $\quad \mathrm{D} p$-brane embeddings}

We study the dynamics of a $\mathrm{D} p$-brane in the $\mathrm{D} q$ background. In the limit $N_{c} \gg 1$ the $\mathrm{D} p$-brane is introduced into the $\mathrm{D} q$ background as a probe, which does not affect the background geometry. The dynamics of the probe $\mathrm{D} p$-brane in the background (3.10) is described by the Dirac-Born-Infeld (DBI) action

$$
S_{\mathrm{D} p}=-T_{p} \int d^{p+1} x e^{-\phi} \sqrt{-\operatorname{det} g_{M N}},
$$

where $g_{M N}(M, N=0,1, \cdots, p)$ is the induced metric on the world-volume and $T_{p}$ is the tension of the $\mathrm{D} p$-brane. For simplicity we have ignored the gauge field on the probe $\mathrm{D} p$-brane.

We use a physical gauge for $\mathrm{D} p$-brane world-volume reparametrizations and use the spacetime coordinates $x^{\mu}(\mu=0,1, \cdots, s), \lambda, \Omega_{p-s-1}$ as the world-volume coordinates. Then the configurations of the $\mathrm{D} p$-brane are determined by $x^{i}(i=$ $s+1, \cdots, q), r$ and $\Omega_{8-q-p+s}$ as a function of those world-volume coordinates. We make an ansatz

$$
x^{s+1}, \cdots, x^{q}=\mathrm{constant}, \quad r=r(\lambda), \quad \theta^{a}=\mathrm{constant},
$$

where $\theta^{a}(a=1,2, \cdots, 8-q-p+s)$ are coordinates of $\mathrm{S}^{8-q-p+s}$.

With this ansatz, the induced metric on the $\mathrm{D} p$-brane is

$$
d s^{2}=\left(\frac{U}{R}\right)^{\frac{7-q}{2}} \eta_{\mu \nu} d x^{\mu} d x^{\nu}+K(\rho)\left[\left(1+\left(r^{\prime}\right)^{2}\right) d \lambda^{2}+\lambda^{2} d \Omega_{p-s-1}^{2}\right],
$$

where $r^{\prime}=\frac{d r}{d \lambda}$. Then the DBI action of the D $p$-brane becomes

$$
S_{\mathrm{D} p}=-\tilde{T}_{p} V_{p-s-1} \int d^{s+1} x \int d \lambda \rho^{\alpha}\left(1+\frac{U_{K K}^{7-q}}{4 \rho^{7-q}}\right)^{\beta} \lambda^{p-s-1} \sqrt{1+\left(r^{\prime}\right)^{2}},
$$

where $\tilde{T}_{p} \equiv g_{s}^{-1} T_{p} R^{-\alpha}$ and $V_{p-s-1}$ is the volume of $\mathrm{S}^{p-s-1}$. The parameters $\alpha$ and $\beta$ are defined as

$$
\alpha=\frac{1}{4}(7-q)(4+2 s-q-p), \quad \beta=\frac{1}{2}(4+2 s-q-p)+\frac{2(p-s)}{7-q} .
$$

The action (3.14) leads to the equation of motion for $r(\lambda)$

$$
\frac{d}{d \lambda}\left[\rho^{\alpha}\left(1+\frac{U_{K K}^{7-q}}{4 \rho^{7-q}}\right)^{\beta} \frac{\lambda^{p-s-1} r^{\prime}}{\sqrt{1+\left(r^{\prime}\right)^{2}}}\right]=\frac{\partial}{\partial r}\left[\rho^{\alpha}\left(1+\frac{U_{K K}^{7-q}}{4 \rho^{7-q}}\right)^{\beta}\right] \lambda^{p-s-1} \sqrt{1+\left(r^{\prime}\right)^{2}} .
$$


As in refs. [7, 8] we are interested in the situation in which the asymptotic distance between the $\mathrm{D} q$-branes and the $\mathrm{D} p$-brane is a finite constant $r_{\infty}$. This constant is proportional to the quark mass. Therefore we impose the boundary conditions for $\lambda \rightarrow \infty$

$$
\left.r(\lambda)\right|_{\lambda \rightarrow \infty}=r_{\infty},\left.\quad r^{\prime}(\lambda)\right|_{\lambda \rightarrow \infty}=0
$$

Then, eq.(3.16) can be linearized at large $\lambda$ as

$$
\frac{d}{d \lambda}\left(\lambda^{\alpha+p-s-1} r^{\prime}\right)=\alpha \lambda^{\alpha+p-s-3} r
$$

and the asymptotic behavior of the solution is

$$
r(\lambda) \sim a \lambda^{k_{+}}+b \lambda^{k_{-}}
$$

where $a, b$ are constants and

$$
k_{ \pm}=\frac{-(\alpha+p-s-2) \pm \sqrt{(\alpha+p-s-2)^{2}+4 \alpha}}{2} .
$$

For the boundary condition (3.17) to be satisfied, we must require $\alpha=0$ and $p-s-2>0$. The first condition implies that the ground states of the NS sector of $q-p$ strings are massless since $\alpha=-2(7-q) a^{\mathrm{NS}}$ as seen from eq. (2.3). Then, the asymptotic behavior of $r(\lambda)$ is

$$
r(\lambda) \sim r_{\infty}+c \lambda^{-(p-s-2)}
$$

where $c$ is a constant. As in ref. [8] the quark condensate $\langle\bar{\psi} \psi\rangle$ can be calculated by differentiating the vacuum energy density derived from the DBI action (3.14) with respect to the quark mass $m_{q}$. Thus we obtain the quark mass and the quark condensate in terms of the constants $r_{\infty}$ and $c$ as

$$
m_{q}=\frac{r_{\infty}}{2 \pi \ell_{s}^{2}}, \quad\langle\bar{\psi} \psi\rangle=-2 \pi(p-s-2) \ell_{s}^{2} \tilde{T}_{p} V_{p-s-1} c
$$

We have numerically solved eq. (3.16) for all possible values of $q, p, s$ satisfying $\alpha=0, p-s-2>0, s \leq 3$. The solutions of the D2/D4 model with $s=1$ and the D3/D5 model with $s=2$ are plotted in Fig. 1 for various values of $r_{\infty}$. The variables $\lambda$ and $r$ in these figures denote dimensionless ones rescaled by appropriate powers of $U_{K K}$. The leftmost curve in these figures represents $U=U_{K K}$. Its interior $U<U_{K K}$ is not a part of the space that we are considering. All the solutions have 


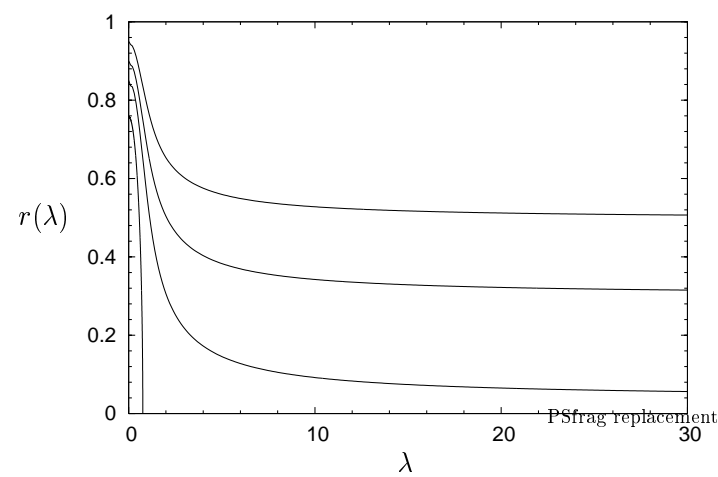

(a)

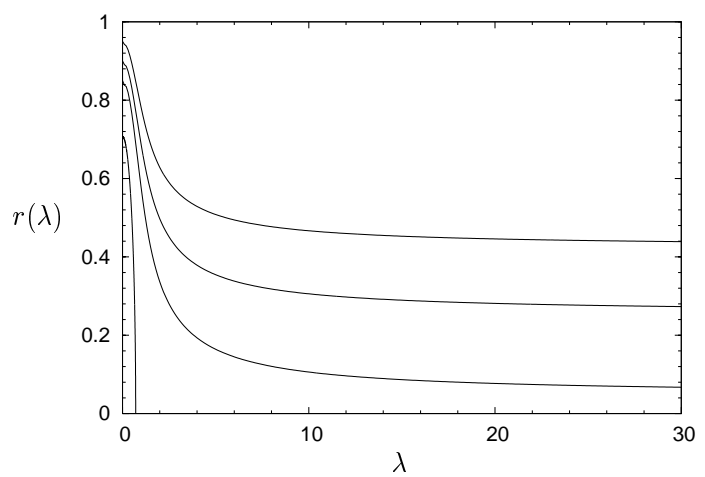

(b)

Figure 1: Solutions of eq. (3.16) for various values of $r_{\infty}$ in (a) the D2/D4 model with $s=1$ and (b) the D3/D5 model with $s=2$.

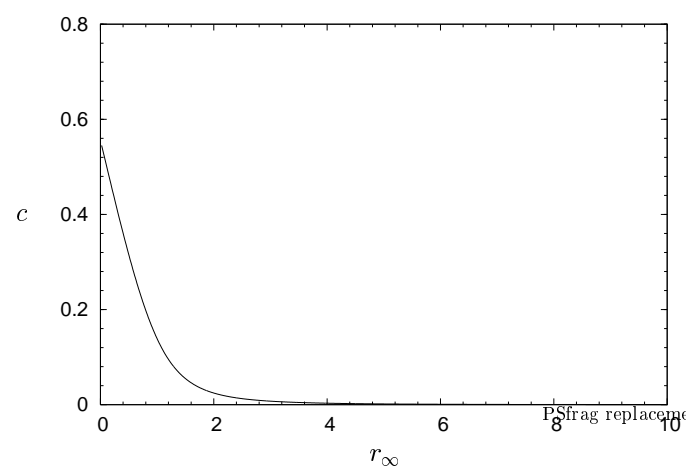

(a)

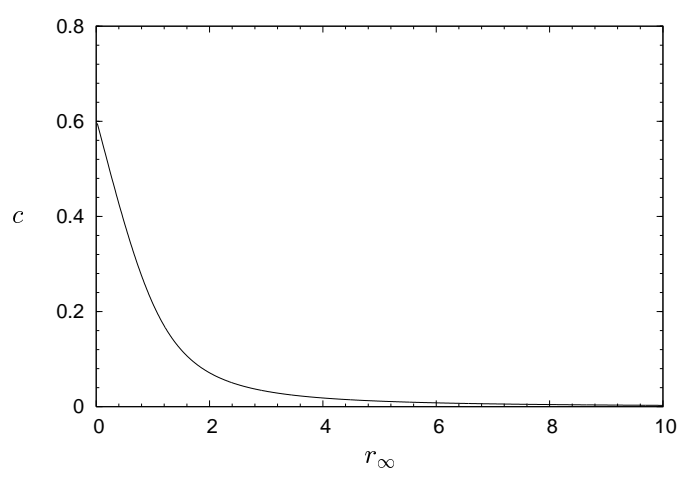

(b)

Figure 2: The quark condensate as a function of the quark mass for (a) the D2/D4 model with $s=1$ and (b) the D3/D5 model with $s=2$.

similar behaviors to those of the D4/D6 model with $s=3$, which was studied in ref. [8]. The solutions approach a constant value $r_{\infty}$ for $\lambda=\infty$, while they reach a point outside of the curve $U=U_{K K}$ at $\lambda=0$. The solutions break the rotational symmetry $\mathrm{SO}(9-q-p+s)$ in the $\left(r, \Omega_{8-q-p+s}\right)$ space to $\mathrm{SO}(8-q-p+s)$.

We have also numerically calculated the quark condensate as a function of the quark mass $c=c\left(r_{\infty}\right)$ for all possible values of $q, p, s$ satisfying $\alpha=0, p-s-2>0$, $s \leq 3$. It is plotted in Fig. 2 for the D2/D4 model with $s=1$ and the D3/D5 model with $s=2$. The variables $r_{\infty}$ and $c$ in these figures denote dimensionless ones rescaled by appropriate powers of $U_{K K}$. For all cases we find a non-zero quark 
condensate for $r_{\infty}=0$. This agrees with a field theoretical view point. In QCD we expect that the chiral symmetry is spontaneously broken by the non-zero quark condensate even for $m_{q}=0$.

Finally, we write down a pattern of the symmetry breaking explicitly. For the class $(q+p, s)=(6,1)$ it is

$$
\mathrm{SU}(2)_{L} \times \mathrm{SU}(2)_{R} \times \mathrm{U}(1)_{V} \rightarrow \mathrm{SU}(2)_{V} \times \mathrm{U}(1)_{V}
$$

for the class $(8,2)$

$$
\mathrm{SU}(2) \times \mathrm{U}(1) \rightarrow \mathrm{U}(1) \times \mathrm{U}(1),
$$

and for the class $(10,3)[8$

$$
\mathrm{U}(1)_{A} \times \mathrm{U}(1)_{V} \rightarrow \mathrm{U}(1)_{V}
$$

\section{NG bosons as fluctuations of the probe brane}

In this section we study fluctuations of the probe brane around the vacuum embedding. In the previous section we have seen that the vacuum embedding breaks $\mathrm{SO}(9-q-p+s)$ rotational symmetry in the $\left(r, \Omega_{8-q-p+s}\right)$ space to $\mathrm{SO}(8-q-p+s)$. This symmetry breaking corresponds to the chiral symmetry breaking for certain sets of $(q+p, s)$. Therefore, there should be $(8-q-p+s)$ Nambu-Goldstone (NG) bosons associated with the symmetry breaking. If quarks are massless these bosons are massless NG bosons. On the other hand, if quarks are massive these are pseudoNG bosons with a non-vanishing mass. We will show that these pseudo-NG bosons satisfy the Gell-Mann-Oakes-Renner (GMOR) relation for a small quark mass $m_{q}$. We will also give the effective action of the fluctuations at quartic order. These results are a generalization of those of the D4/D6 system studied in ref. [8] to the $\mathrm{D} q / \mathrm{D} p$ systems.

\subsection{Fluctuations around the vacuum embeddings}

We study fluctuation modes around the vacuum $\mathrm{D} p$-brane embedding

$$
x^{s+1}, \cdots, x^{q}=\text { constant }, \quad r=r_{\mathrm{vac}}(\lambda), \quad \theta^{a}=0+\delta \theta^{a}\left(x^{M}\right),
$$


where $r_{\text {vac }}$ is the vacuum embedding determined numerically in the previous section. For simplicity we concentrate on fluctuations of $\theta^{a}$. In general, these fluctuations depend on all of the world-volume coordinates $x^{M}$ of the $\mathrm{D} p$-brane. We will see that the fluctuations $\delta \theta^{a}$ are identified with the (pseudo-)NG bosons for the breaking of the rotational symmetry of $\mathrm{S}^{8-q-p+s}$ (a subspace of the $\left(r, \Omega_{8-q-p+s}\right)$ space).

The induced metric on the Dp-brane world-volume is

$$
\begin{gathered}
d s^{2}=\left(\frac{U}{R}\right)^{\frac{7-q}{2}} \eta_{\mu \nu} d x^{\mu} d x^{\nu}+K(\rho)\left[\left(1+\left(r_{\text {vac }}^{\prime}\right)^{2}\right) d \lambda^{2}+\lambda^{2} d \Omega_{p-s-1}^{2}\right] \\
+K(\rho) r_{\text {vac }}^{2} \gamma_{a b} \partial_{M} \delta \theta^{a} \partial_{N} \delta \theta^{b} d x^{M} d x^{N}
\end{gathered}
$$

where $\rho^{2}=\lambda^{2}+r_{\text {vac }}^{2}$ and $\gamma_{a b}$ is the metric of a unit $\mathrm{S}^{8-q-p+s}$. Then the DBI action of the D $p$-brane (3.11) to quadratic order becomes

$$
S_{\mathrm{D} p}=S_{\mathrm{vac}}+S_{\delta \theta}
$$

where $S_{\text {vac }}$ is the action for the vacuum embedding, i.e., eq. (3.14) for $r=r_{\text {vac }}$, and $S_{\delta \theta}$ is the action for the fluctuations $\delta \theta^{a}$. After some simple calculations we obtain the action for $\delta \theta^{a}$

$$
\begin{array}{rl}
S_{\delta \theta}=-\tilde{T}_{p} \int d^{p+1} & x \sqrt{\operatorname{det} \gamma_{\alpha \beta}} \lambda^{p-s-1} \sqrt{1+\left(r_{\mathrm{vac}}^{\prime}\right)^{2}} \\
& \times\left(1+\frac{U_{K K}^{7-q}}{4 \rho_{\mathrm{vac}}^{7-q}}\right)^{\beta} \frac{K}{2} g^{M N} r_{\mathrm{vac}}^{2} \gamma_{a b} \partial_{M} \delta \theta^{a} \partial_{N} \delta \theta^{b}
\end{array}
$$

and the equation of motion

$$
\begin{aligned}
\left(\frac{7-q}{2}\right)^{2} & \frac{U_{K K}^{5-q}}{M_{K K}^{2}} \rho_{\mathrm{vac}}^{-(7-q)}\left(1+\frac{U_{K K}^{7-q}}{4 \rho_{\mathrm{vac}}^{7-q}}\right)^{\beta-\frac{2(5-q)}{7-q}} r_{\mathrm{vac}}^{2} \partial_{\mu} \partial^{\mu} \delta \theta^{a} \\
& +\frac{1}{\lambda^{p-s-1} \sqrt{1+\left(r_{\mathrm{vac}}^{\prime}\right)^{2}}} \frac{\partial}{\partial \lambda}\left[\left(1+\frac{U_{K K}^{7-q}}{4 \rho_{\mathrm{vac}}^{7-q}}\right)^{\beta} \frac{\lambda^{p-s-1} r_{\mathrm{vac}}^{2}}{\sqrt{1+\left(r_{\mathrm{vac}}^{\prime}\right)^{2}}} \frac{\partial}{\partial \lambda} \delta \theta^{a}\right] \\
& +\left(1+\frac{U_{K K}^{7-q}}{4 \rho_{\mathrm{vac}}^{7-q}}\right)^{\beta} \frac{r_{\mathrm{vac}}^{2}}{\lambda^{2}} \nabla^{2} \delta \theta^{a}=0,
\end{aligned}
$$

where $\gamma_{\alpha \beta}$ and $\nabla^{2}$ are the metric and the Laplacian on a unit $\mathrm{S}^{p-s-1}$.

We can write a solution of the equation of motion (4.5) in a form

$$
\delta \theta^{a}=F^{a}(\lambda) Y\left(\Omega_{p-s-1}\right) e^{i k \cdot x}
$$


where $Y\left(\Omega_{p-s-1}\right)$ is the spherical harmonics on $\mathrm{S}^{p-s-1}$. We consider the zero (constant) mode of $Y$ and study only lowest-mass modes for simplicity. Substituting eq. (4.6) into eq. (4.5) we obtain an eigenvalue equation for the $(s+1)$-dimensional mass $M_{\theta}^{2}=-k^{\mu} k_{\mu}$. Although we can solve eq. (4.5) by numerical calculations as in ref. [8], here we are content with asymptotic solutions of a linearized equation of motion for $\lambda \rightarrow \infty$. Taking account of the asymptotic behavior of $r_{\text {vac }}$ in eq. (3.21) the first term of eq. (4.5) is sub-leading and the linearized equation for $\lambda \rightarrow \infty$ becomes

$$
\frac{\partial}{\partial \lambda}\left(\lambda^{p-s-1} r_{\operatorname{vac}}^{2} \frac{\partial}{\partial \lambda} \delta \theta^{a}\right)=0 .
$$

Depending on the value $r_{\infty}$ in eq. (3.21) the general solution is

$$
\delta \theta^{a} \sim \begin{cases}a \lambda^{p-s-2}+b & \left(r_{\infty}=0\right) \\ a+b \lambda^{-(p-s-2)} & \left(r_{\infty} \neq 0\right),\end{cases}
$$

where $a, b$ are independent of $\lambda$. Since $p-s-2>0$, these solutions are normalizable when $a=0$.

The non-linear equation of motion (4.5) has exact solutions $\delta \theta^{a}=F^{a} e^{i k \cdot x}\left(F^{a}=\right.$ constant), which have an eigenvalue $M_{\theta}=0$. From the above results on the asymptotic behaviors these solutions are normalizable only when $r_{\infty}=0$. Since $r_{\infty}=0$ means vanishing quark mass $m_{q}=0$, the normalizable solutions with $M_{\theta}=0$ can be regarded as the NG bosons associated with the spontaneous breaking of the rotational symmetry $\mathrm{SO}(9-q-p+s)$. When $r_{\infty} \neq 0\left(m_{q} \neq 0\right)$, the quark mass term explicitly breaks the chiral symmetry and we do not expect massless bosons. However, for small quark mass $m_{q}$ there should exist pseudo-NG bosons with a small mass $M_{\theta}$, which we consider in the next subsection.

In two dimensions there exists no massless NG boson associated with a spontaneous symmetry breaking [41]. We have seen that there appear massless bosons even in the D2/D4 model with a one-dimensional intersection when quarks are massless. These massless bosons should be an artifact of the large $N_{c}$ limit and should become massive if we take into account contributions from higher orders in the $1 / N_{c}$ expansion. The situation is similar to the case of the Gross-Neveu model in two dimensions [42], in which massless bosons appear in the large $N$ limit.

\subsection{Light pseudo-NG bosons and the GMOR relation}

As we have seen above, the embeddings with $r_{\infty}=0$ and those with $r_{\infty} \neq 0$ have different properties. For the $r_{\infty}=0$ embeddings the asymptotic distance between 
$\mathrm{D} q$ and $\mathrm{D} p$-branes is zero and the quarks at the intersection are massless. There are $(8-q-p+s)$ massless scalars $\delta \theta^{a}$ in the spectrum, which can be identified with the NG bosons associated with the spontaneous symmetry breaking $\mathrm{SO}(9-q-p+s)$ $\rightarrow \mathrm{SO}(8-q-p+s)$. We call these NG bosons pions. On the other hand, for the $r_{\infty} \neq 0$ embeddings quarks are massive and the vacuum embedding explicitly breaks the rotational symmetry $\mathrm{SO}(9-q-p+s)$ even for the asymptotic region $\lambda \rightarrow \infty$. In this case the fluctuations $\delta \theta^{a}$ are pseudo-NG bosons with a non-vanishing mass $M_{\theta}$.

We can show the Gell-Mann-Oakes-Renner (GMOR) relation [38]

$$
M_{\theta}^{2}=-\frac{m_{q}\langle\bar{\psi} \psi\rangle}{f_{\pi}^{2}}
$$

for a small quark mass $m_{q}$ by using the holographic method [8]. Here, $f_{\pi}$ is the pion decay constant. We begin with the $r_{\infty}=0$ embedding and make a small change $r_{\infty}=\delta r_{\infty}$. This gives a small mass to quarks. As shown in ref. [8] the mass of the pseudo-NG bosons $M_{\theta}$ can be obtained by using a standard perturbation theory in quantum mechanics and is written as

$$
M_{\theta}^{2}=(p-s-2) \frac{\bar{c} \delta r_{\infty}}{\int d \lambda \bar{\mu}}
$$

where $\bar{c}$ is the coefficient in eq. (3.21) and $\bar{\mu}$ is given by

$$
\bar{\mu}=\left(\frac{7-q}{2}\right)^{2} \frac{U_{K K}^{5-q}}{M_{K K}^{2}} \bar{\rho}_{\mathrm{vac}}^{-(7-q)}\left(1+\frac{U_{K K}^{7-q}}{4 \bar{\rho}_{\mathrm{vac}}^{7-q}}\right)^{\beta-\frac{2(5-q)}{7-q}} \bar{r}_{\mathrm{vac}}^{2} \lambda^{p-s-1} \sqrt{1+\left(\bar{r}_{\mathrm{vac}}^{\prime}\right)^{2}} .
$$

Here and in the following, quantities with a bar denote those for the $r_{\infty}=0$ embedding. The quantities $\delta r_{\infty}$ and $\bar{c}$ are related to the quark mass and the quark condensate as in eq. (3.22). The pion decay constant $f_{\pi}$ can be read from the effective action of $\delta \theta^{a}$. Assuming that $\delta \theta^{a}$ depend only on the coordinates of the intersection $x^{\mu}(\mu=0,1, \cdots, s)$ and integrating over $\lambda$ and $\Omega_{p-s-1}$ in eq. (4.4) we obtain

$$
S_{\delta \theta}=-f_{\pi}^{2} \int d^{s+1} x \frac{1}{2} \gamma_{a b} \partial_{\mu} \delta \theta^{a} \partial^{\mu} \delta \theta^{b}
$$

where $f_{\pi}$ is given by

$$
f_{\pi}^{2}=\tilde{T}_{p} V_{p-s-1} \int_{0}^{\infty} d \lambda \bar{\mu}
$$

Using eqs. (3.22), (4.13) in eq. (4.10), we obtain the GMOR relation (4.9). 


\subsection{The pion effective action}

We can write down the effective action of the pion fields $\delta \theta^{a}$ at the intersection beyond the quadratic order. We assume that $\delta \theta^{a}$ depend only on the coordinates of the intersection $x^{\mu}(\mu=0,1, \cdots, s)$. By expanding the DBI action (3.11) for the induced metric (4.2) to quartic order in $\delta \theta^{a}$ we obtain

$$
\begin{aligned}
S_{\delta \theta}= & -\int d^{s+1} x\left(\frac{f_{\pi}^{2}}{2} \gamma_{a b} \partial_{\mu}\left(\delta \theta^{a}\right) \partial^{\mu}\left(\delta \theta^{b}\right)+\frac{h_{1}}{4}\left[\gamma_{a b} \partial_{\mu}\left(\delta \theta^{a}\right) \partial^{\mu}\left(\delta \theta^{b}\right)\right]^{2}\right. \\
& \left.-\frac{h_{2}}{4}\left[\gamma_{a b} \partial_{\mu}\left(\delta \theta^{a}\right) \partial_{\nu}\left(\delta \theta^{b}\right)\right]\left[\gamma_{c d} \partial^{\mu}\left(\delta \theta^{c}\right) \partial^{\nu}\left(\delta \theta^{d}\right)\right]\right)
\end{aligned}
$$

where $f_{\pi}$ is the pion decay constant (4.13) and the constants $h_{1}, h_{2}$ are given by

$$
\begin{aligned}
2 h_{1}=h_{2}= & \tilde{T}_{p} V_{p-s-1}\left(\frac{7-q}{2}\right)^{4} \frac{U_{K K}^{2(5-q)}}{M_{K K}^{4}} \int d \lambda \rho_{\text {vac }}^{-2(7-q)} \\
& \times\left(1+\frac{U_{K K}^{7-q}}{4 \rho_{\text {vac }}^{7-q}}\right)^{\beta-\frac{4(5-q)}{7-q}} r_{\text {vac }}^{4} \lambda^{p-s-1} \sqrt{1+\left(r_{\text {vac }}^{\prime}\right)^{2}} .
\end{aligned}
$$

The relative coefficients of the quartic terms are different from those assumed in the Skyrme model [43, 44] $h_{1}=h_{2}$. This is in contrast with another approach [13] to the holographic QCD, in which the relation $h_{1}=h_{2}$ of the Skyrme model was obtained.

\section{5. $s=q$ configurations}

In this section we study a special case $s=q$ of the configuration (2.1)

\begin{tabular}{rccccccccc} 
& $x^{0}$ & $\cdots$ & $x^{q}$ & $x^{q+1}$ & $\cdots$ & $x^{p}$ & $x^{p+1}$ & $\cdots$ & $x^{9}$ \\
\hline$N_{c} \mathrm{D} q$ & $\circ$ & $\cdots$ & $\circ$ & - & $\cdots$ & - & - & $\cdots$ & - \\
$\mathrm{D} p$ & $\circ$ & $\cdots$ & $\circ$ & $\circ$ & $\cdots$ & $\circ$ & - & $\cdots$ & -
\end{tabular}

with $x^{q}$ being a coordinate of $\mathrm{S}^{1}$. It has a $q$-dimensional intersection along the $x^{1}, \cdots, x^{q}$ directions. The global symmetry preserved at the intersection contains

$$
\mathrm{SO}(1, q-1) \times \mathrm{SO}(9-p) \times \mathrm{U}(1) .
$$




\begin{tabular}{|c|c|cccccccccc|}
\hline & & 0 & 1 & 2 & 3 & 4 & 5 & 6 & 7 & 8 & 9 \\
\hline color & D1 & $\circ$ & $\circ$ & - & - & - & - & - & - & - & - \\
\hline probe & D5 & $\circ$ & $\circ$ & $\circ$ & $\circ$ & $\circ$ & $\circ$ & - & - & - & - \\
\hline \hline color & D2 & $\circ$ & $\circ$ & $\circ$ & - & - & - & - & - & - & - \\
\hline probe & D6 & $\circ$ & $\circ$ & $\circ$ & $\circ$ & $\circ$ & $\circ$ & $\circ$ & - & - & - \\
\hline \hline color & D3 & $\circ$ & $\circ$ & $\circ$ & $\circ$ & - & - & - & - & - & - \\
\hline probe & D7 & $\circ$ & $\circ$ & $\circ$ & $\circ$ & $\circ$ & $\circ$ & $\circ$ & $\circ$ & - & - \\
\hline
\end{tabular}

Table 2: $\mathrm{D} q / \mathrm{D} p$ brane configurations with a $q$-dimensional intersection dual to $\mathrm{QCD}_{q}$.

Note that the Lorentz symmetry at the intersection is $\mathrm{SO}(1, q-1)$ in the present case since one of the spatial directions $x^{q}$ is compactified on $\mathrm{S}^{1}$. $\mathrm{SO}(9-p)$ and $\mathrm{U}(1)$ are the same as in eq. (2.2).

As in sect. 3 we only consider the cases in which the asymptotic distance between the $\mathrm{D} q$-branes and the $\mathrm{D} p$-brane is a constant $r_{\infty}$. This will require $\# \mathrm{ND}=4$ and $p-q-2>0$. The only configurations satisfying these two conditions and $s \leq 3$ are the D1/D5 model with $s=1$, the D2/D6 model with $s=2$ and the D3/D7 model with $s=3$. They are listed in Table 2, The theory localized at the intersection is $\mathrm{QCD}_{q+1}$ on $\mathbb{R}^{1, q-1} \times \mathrm{S}^{1}$, which can be regarded as $\mathrm{QCD}_{q}$ on $\mathbb{R}^{1, q-1}$ at sufficiently low energy.

Note that the $\mathrm{SO}(9-p)$ rotational symmetry in the transverse space cannot be regarded as the chiral symmetry of $\mathrm{QCD}_{q}$ in the present case because of the dimensional reduction from $\mathrm{QCD}_{q+1}$ to $\mathrm{QCD}_{q}$. For $\mathrm{QCD}_{1}$ from the D1/D5 model with $s=1$ there is an $\mathrm{SU}(2) \times \mathrm{SU}(2)$ symmetry from the rotational symmetry $\mathrm{SO}(4)_{6789}$ of the transverse space but there is no chirality in one dimension. For $\mathrm{QCD}_{2}$ from the D2/D6 model with $s=2$ there exist two massless fermions of positive and negative chiralities. The $\mathrm{SU}(2)$ symmetry from the rotational symmetry $\mathrm{SO}(3)_{789}$ acts on both of them in the same way and therefore is not a chiral symmetry. For $\mathrm{QCD}_{3}$ from the D3/D7 model with $s=3$ there is a $\mathrm{U}(1)$ symmetry from the rotational symmetry $\mathrm{SO}(2)_{89}$ but there is no chirality in three-dimensional spacetime. In the following we study a spontaneous breaking of these symmetries by numerically calculating configurations of the $\mathrm{D} p$-brane embeddings and the quark mass dependence of the condensate. 
In the present case the induced metric on the $\mathrm{D} p$-brane is

$$
\begin{array}{r}
d s^{2}=\left(\frac{U}{R}\right)^{\frac{7-q}{2}}\left(-d t^{2}+\sum_{i=1}^{q-1}\left(d x^{i}\right)^{2}+f(U)\left(d x^{q}\right)^{2}\right) \\
+K(\rho)\left[\left(1+\left(r^{\prime}\right)^{2}\right) d \lambda^{2}+\lambda^{2} d \Omega_{p-q-1}^{2}\right] .
\end{array}
$$

Here we have used the same ansatz (3.12) for the embedding as in the previous analysis. There appears an extra factor $f(U)$ in front of $\left(d x^{q}\right)^{2}$ in contrast to the metric (3.13). Then the DBI action of the $\mathrm{D} p$-brane becomes

$$
S_{\mathrm{D} p}=-\tilde{T}_{p} V_{p-q-1} \int d^{q+1} x \int d \lambda \rho^{\alpha}\left(1+\frac{U_{K K}^{7-q}}{4 \rho^{7-q}}\right)^{\beta-1}\left(1-\frac{U_{K K}^{7-q}}{4 \rho^{7-q}}\right) \lambda^{p-q-1} \sqrt{1+\left(r^{\prime}\right)^{2}},
$$

where $\alpha$ and $\beta$ are given by eq. (3.15) with $s=q$. The action (5.4) leads to the equation of motion for $r(\lambda)$

$$
\begin{gathered}
\frac{d}{d \lambda}\left[\rho^{\alpha}\left(1+\frac{U_{K K}^{7-q}}{4 \rho^{7-q}}\right)^{\beta-1}\left(1-\frac{U_{K K}^{7-q}}{4 \rho^{7-q}}\right) \frac{\lambda^{p-q-1} r^{\prime}}{\sqrt{1+\left(r^{\prime}\right)^{2}}}\right] \\
=\frac{\partial}{\partial r}\left[\rho^{\alpha}\left(1+\frac{U_{K K}^{7-q}}{4 \rho^{7-q}}\right)^{\beta-1}\left(1-\frac{U_{K K}^{7-q}}{4 \rho^{7-q}}\right)\right] \lambda^{p-q-1} \sqrt{1+\left(r^{\prime}\right)^{2}}
\end{gathered}
$$

As in sect. 3 the boundary conditions $\left.r(\lambda)\right|_{\lambda \rightarrow \infty}=r_{\infty}$ and $\left.r^{\prime}(\lambda)\right|_{\lambda \rightarrow \infty}=0$ require $\alpha=0$ and $p-q-2>0$. Then, the asymptotic behavior of the general solution $r(\lambda)$ has a form in eq. (3.21) with the coefficients related to the quark mass $m_{q}$ and the condensate $\langle\bar{\psi} \psi\rangle$ as in eq. (3.22).

We can solve the equation of motion (5.5) by numerical calculations. The D3/D7 model was studied in ref. [7]. Solutions for other models in Table 2 are plotted in Fig. 3 for various values of $r_{\infty}$. The variables in Fig. 3 (and Fig. 4 below) denote dimensionless ones rescaled by appropriate powers of $U_{K K}$. These solutions have similar behaviors to that of the D3/D7 model [7]. Note that two types of embeddings are possible in contrast to the cases in sect. 3. One type of embeddings, which are possible for sufficiently large $r_{\infty}$, do not reach the curve $U=U_{K K}$ similarly to the embeddings in sect. 3. The other type of embeddings, which are possible for sufficiently small $r_{\infty}$, reach the curve $U=U_{K K}$. Such embeddings do not exist in the models discussed in sect. 3. In particular, the solution for $r_{\infty}=0$ is $r(\lambda)=0$, which preserves the rotational symmetry $\mathrm{SO}(9-p)$ in the transverse directions. For an intermediate region of $r_{\infty}$ more than one embeddings, which can be either type 


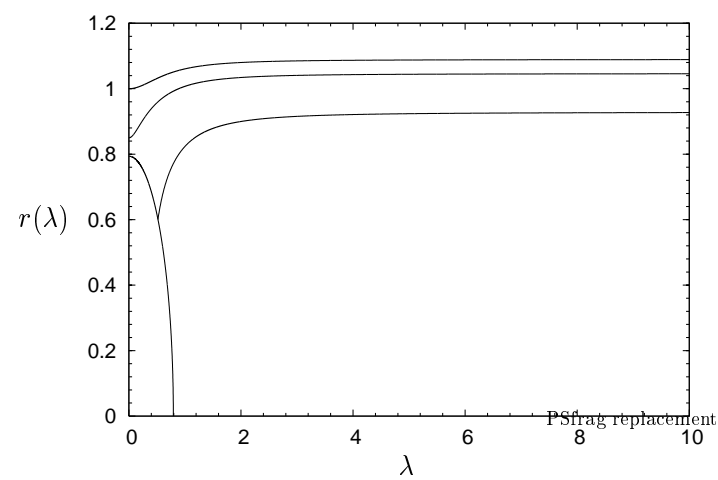

(a)

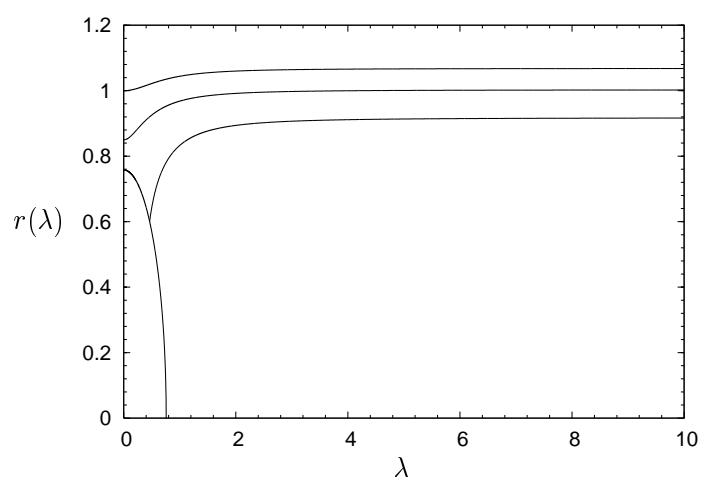

(b)

Figure 3: Solutions of eq. (5.5) for various values of $r_{\infty}$ in (a) the D1/D5 model with $s=1$ and (b) the D2/D6 model with $s=2$.

of embeddings, are possible. The physically realized embedding is the one with a minimal energy.

We have also numerically calculated the quark condensate as a function of the quark mass $c=c\left(r_{\infty}\right)$ and plotted them in Fig. 4. For all the cases the quark condensate vanishes at $r_{\infty}=0$. This is consistent with the restoration of the symmetry $\mathrm{SO}(9-p)$ in the massless quark limit, which was found above from the solution of the embedding equation for $r_{\infty}=0$. Therefore we do not expect massless NG bosons to appear. Indeed, in the case of the D3/D7 model, it was shown [7] that the meson spectrum has a mass gap by numerically solving an equation of motion for the probe brane fluctuations.

\section{Finite temperature analysis}

To study the theory at finite temperature we introduce a periodic Euclidean time coordinate $t_{E} \equiv i t \sim t_{E}+\delta t_{E}$. The period of $t_{E}$ is the inverse temperature $\delta t_{E}=1 / T$. Then there are two periodic coordinates $t_{E}$ and $x^{q}$. There exist two possible Euclidean geometries which have an appropriate asymptotic behavior. One of them is the Euclidean version of eq. (3.1). The other is the Euclidean version of 


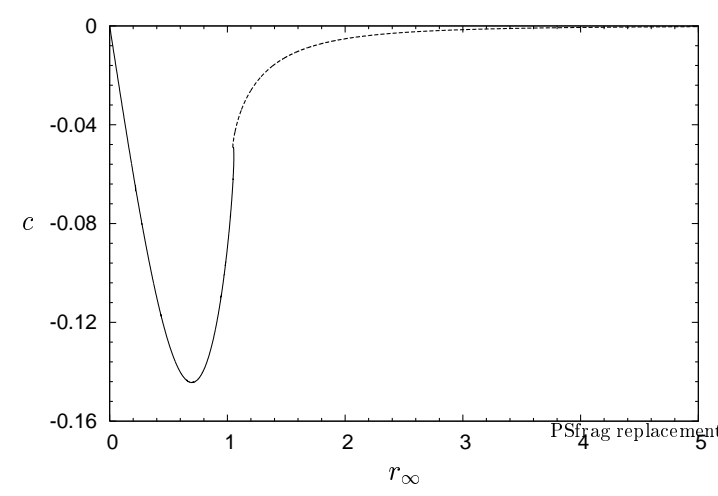

(a)

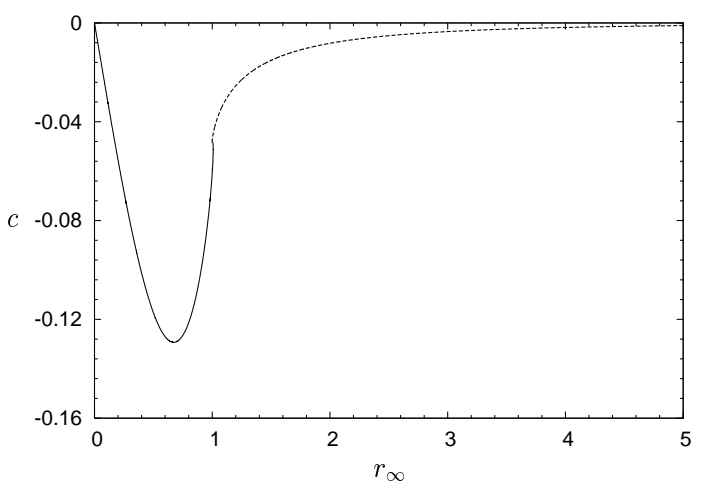

(b)

Figure 4: The quark condensate as a function of the quark mass for (a) the D1/D5 model with $s=1$ and (b) the D2/D6 model with $s=2$. The solid (dashed) lines represent the contributions from the embeddings which do (not) reach the curve $U=U_{K K}$.

the non-extremal black D $q$-brane geometry

$$
\begin{gathered}
d s^{2}=\left(\frac{U}{R}\right)^{\frac{7-q}{2}}\left(\tilde{f}(U) d t_{E}^{2}+\sum_{i=1}^{q-1}\left(d x^{i}\right)^{2}+\left(d x^{q}\right)^{2}\right)+\left(\frac{R}{U}\right)^{\frac{7-q}{2}}\left(\frac{d U^{2}}{\tilde{f}(U)}+U^{2} d \Omega_{8-q}^{2}\right) \\
\tilde{f}(U)=1-\left(\frac{U_{T}}{U}\right)^{7-q}
\end{gathered}
$$

with the dilaton and the RR-flux given in eq. (3.3). To avoid a conical singularity at $U=U_{T}$ in the $U-t_{E}$ plane the period $\delta t_{E}$ is fixed as

$$
\delta t_{E}=\frac{4 \pi R^{\frac{7-q}{2}}}{(7-q) U_{T}^{\frac{5-q}{2}}} .
$$

It was shown [45, 8, 27] that the Euclidean version of the background (3.1) is dominant at low temperature, while the background (6.1) is dominant at high temperature by comparing values of the Euclidean supergravity action for these backgrounds. A phase transition occurs at the temperature $T_{\text {deconf }}=M_{K K} /(2 \pi)$. This phase transition corresponds to a confinement/deconfinement transition in the dual gauge theory [45].

We consider the probe brane dynamics in the high temperature phase. The probe brane dynamics in the low temperature phase is essentially the same as at zero temperature. We only consider the models with $\alpha=0, p-s-2>0, s \leq 3$ as 
in the zero temperature phase. With the ansatz (3.12) the induced metric on the $\mathrm{D} p$-brane in the background (6.1) can be written as

$$
d s^{2}=\left(\frac{U}{R}\right)^{\frac{7-q}{2}}\left(\tilde{f}(U) d t_{E}^{2}+\sum_{i=1}^{s}\left(d x^{i}\right)^{2}\right)+K(\rho)\left[\left(1+\left(r^{\prime}\right)^{2}\right) d \lambda^{2}+\lambda^{2} d \Omega_{p-s-1}^{2}\right]
$$

which is valid for $s=q$ as well as for $s<q$. Then the DBI action of the probe $\mathrm{D} p$-brane becomes

$$
S_{\mathrm{D} p}=\tilde{T}_{p} V_{p-s-1} \int d^{s+1} x \int d \lambda\left(1+\frac{U_{T}^{7-q}}{4 \rho^{7-q}}\right)^{\beta-1}\left(1-\frac{U_{T}^{7-q}}{4 \rho^{7-q}}\right) \lambda^{p-s-1} \sqrt{1+\left(r^{\prime}\right)^{2}},
$$

which leads to the equation of motion for $r(\lambda)$

$$
\begin{aligned}
\frac{d}{d \lambda}\left[\left(1+\frac{U_{T}^{7-q}}{4 \rho^{7-q}}\right)^{\beta-1}\right. & \left.\left(1-\frac{U_{T}^{7-q}}{4 \rho^{7-q}}\right) \frac{\lambda^{p-s-1} r^{\prime}}{\sqrt{1+\left(r^{\prime}\right)^{2}}}\right] \\
& =\frac{\partial}{\partial r}\left[\left(1+\frac{U_{T}^{7-q}}{4 \rho^{7-q}}\right)^{\beta-1}\left(1-\frac{U_{T}^{7-q}}{4 \rho^{7-q}}\right)\right] \lambda^{p-s-1} \sqrt{1+\left(r^{\prime}\right)^{2}}
\end{aligned}
$$

Note that the action (6.4) and therefore the equation of motion (6.5) are identical to those of the $s=q$ configuration at zero temperature (5.4) and (5.5) up to an exchange of $U_{T}$ and $U_{K K}$.

The asymptotic behavior of the solution $r(\lambda)$ of eq. (6.5) for large $\lambda$ is the same as in the zero temperature case (3.21). The parameters $r_{\infty}$ and $c$ are related to the quark mass $m_{q}$ and the quark condensate $\langle\bar{\psi} \psi\rangle$ as in eq. (3.22). We have numerically solved eq. (6.5) for all possible values of $q, p, s$. All the solutions have similar behaviors to those for the D3/D7 model with $s=3$ and the D4/D6 model with $s=3$ discussed in refs. [7, 8, 29, 31]. The solutions for the D2/D4 model with $s=1$ and the D3/D5 model with $s=2$ are plotted in Fig. 5 for various values of $r_{\infty}$. The variables $\lambda$ and $r$ in these figures (and Fig. 6 below) denote dimensionless ones rescaled by appropriate powers of $U_{T}$. The leftmost curve in these figures represents $U=U_{T}$.

We have also numerically calculated the quark condensate $c=c\left(r_{\infty}\right)$. Here we are interested in the phase structure of the system when the temperature $T$ is varied for fixed quark mass $m_{q}$. The relation between $T$ and $r_{\infty}$ can be obtained from eqs. 


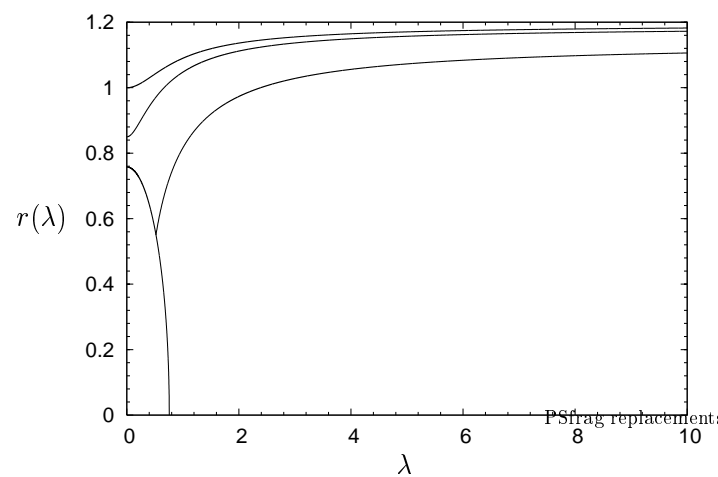

(a)

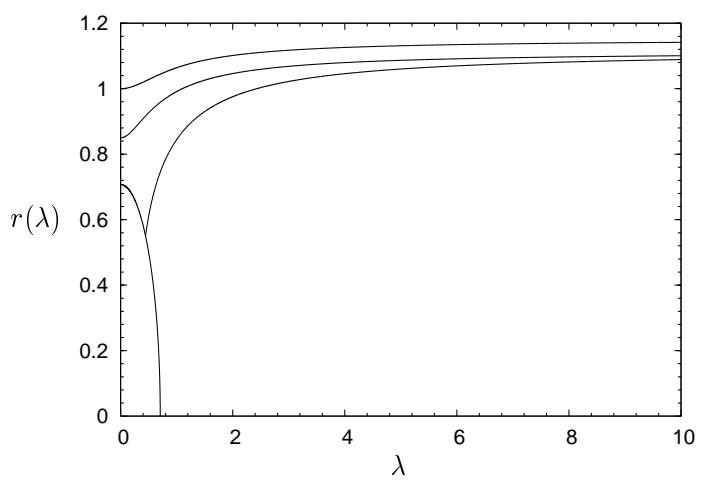

(b)

Figure 5: Solutions of eq. (6.5) for various values of $r_{\infty}$ in (a) the D2/D4 model with $s=1$ and (b) the D3/D5 model with $s=2$.

$(3.2),(3.4),(6.2)$ as

$$
T=\frac{\bar{M}}{\sqrt{r_{\infty}^{5-q}}}, \quad \bar{M}^{2}=\frac{(7-q)^{2} m_{q}^{5-q} M_{K K}}{4(4 \pi)^{\frac{5-q}{2}} \Gamma\left(\frac{7-q}{2}\right) g_{q}^{2} N_{c}},
$$

where $g_{q}=g_{q+1} / \delta x^{q}$ is the $q$-dimensional Yang-Mills coupling and $r_{\infty}$ is the dimensionless variable rescaled by $U_{T}$. Using this relation the quark condensate as a function of the temperature is plotted in Fig. 6. Note that the region near $T=0$ in these figures is not valid since the background (3.1) is dominant at low temperature $T<T_{\text {deconf. }}$ All the condensates have similar behaviors to those of the D3/D7 model with $s=3$ and the D4/D6 model with $s=3$ discussed in refs. [7, 8, 29, 31].

As was discussed in refs. [7, 8, 29, 31] there are two types of embeddings. For sufficiently large $r_{\infty}$ the probe brane does not reach the horizon $U=U_{T}$. On the other hand, for sufficiently small $r_{\infty}$ it reaches the horizon $U=U_{T}$. For an intermediate region of $r_{\infty}$ more than one embeddings, which can be either type of embeddings, are possible. The physically realized embedding is the one with a minimal energy. Varying the value of $r_{\infty}$ a phase transition between these two types of embeddings occurs at a certain temperature $T=T_{\text {fund }}$. This phase transition, however, does not affect the chiral symmetry of the quarks in $\mathrm{QCD}_{s+1}$ because of the non-zero value of $c$ for all temperature region except for $T \rightarrow \infty$.

Finally we note that a chemical potential for the baryon number can be introduced by considering the $\mathrm{U}(1)$ gauge field on the probe $\mathrm{D} p$-brane [32, 33]. An asymptotically non-vanishing Euclidean time component of the U(1) gauge field can 


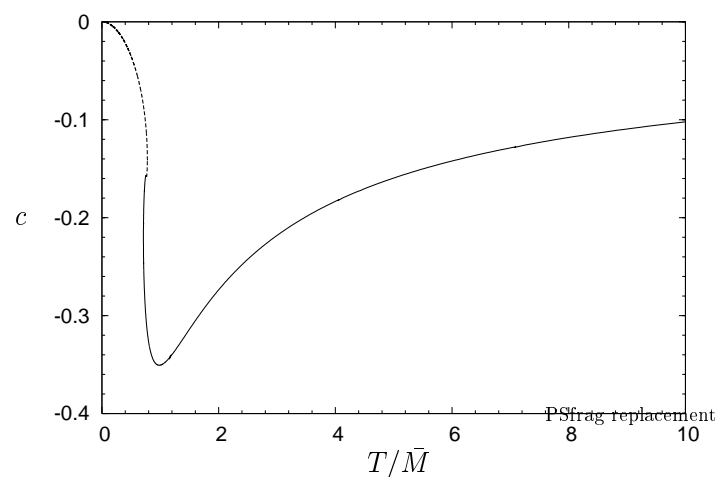

(a)

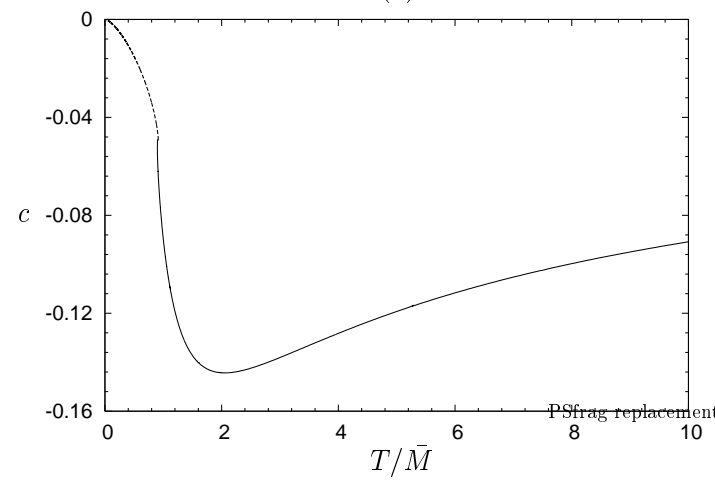

(c)

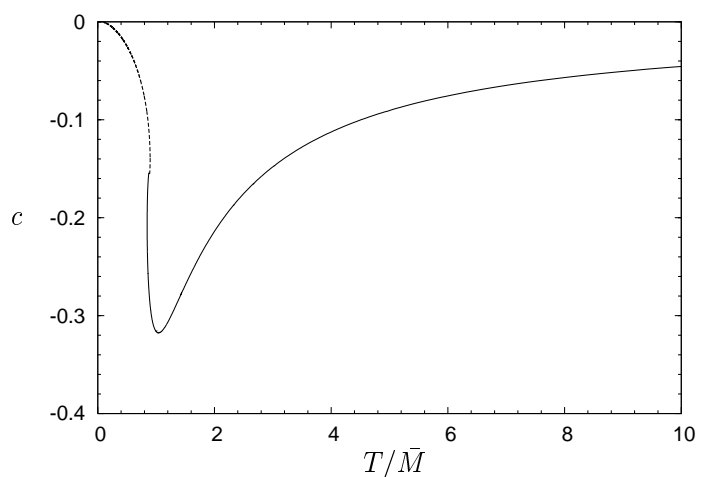

(b)

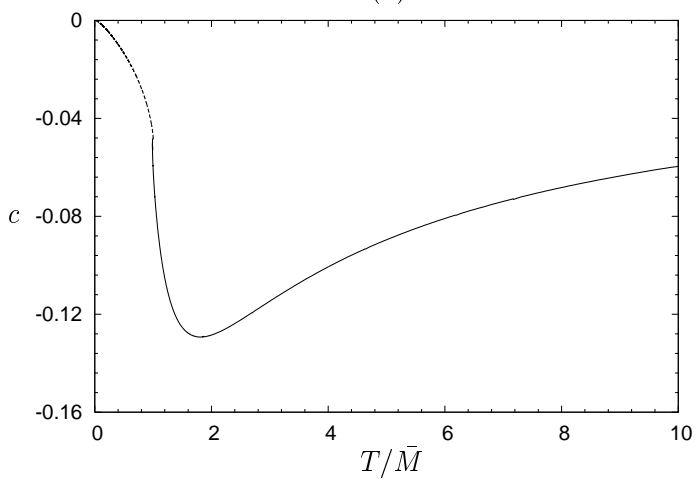

(d)

Figure 6: The quark condensate as a function of temperature for (a) the D2/D4 model with $s=1$, (b) the D3/D5 model with $s=2$, (c) the D1/D5 model with $s=1$ and (d) the D2/D6 model with $s=2$. The solid (dashed) lines represent the contributions from the embeddings which do (not) reach the horizon $U=U_{T}$.

be understood as a chemical potential. It will be possible to discuss the phase diagram in the $(\mu, T)$ space by using this chemical potential as in refs. [36, 37].

\section{Conclusion}

In this paper we discussed the chiral symmetry breaking in the $\mathrm{D} q / \mathrm{D} p$ model with an $s$-dimensional intersection. There exist QCD-like theories at the intersection for certain cases. We are interested in the models which have directions transverse to both of the $\mathrm{D} q$ and $\mathrm{D} p$-branes. The rotational symmetry in these directions can be identified with the chiral symmetry in certain cases. For instance, it is a non-Abelian 
chiral symmetry $\mathrm{SU}(2)_{L} \times \mathrm{SU}(2)_{R}$ in the D2/D4 model with $s=1$ corresponding to $\mathrm{QCD}_{2}$. In these models we studied the dynamics of the probe $\mathrm{D} p$-brane in the $\mathrm{D} q$ background at zero and at finite temperature.

At zero temperature we found that the rotational symmetry in the transverse directions is broken by a $\mathrm{D} p$-brane embedding. This corresponds to the chiral symmetry breaking in $\mathrm{QCD}_{s+1}$. We numerically calculated the quark condensate $\langle\bar{\psi} \psi\rangle$ as a function of the quark mass $m_{q}$. When $s<q$, we found that there is a non-zero quark condensate even for zero quark mass and therefore the symmetry is spontaneously broken. When $s=q$, we found that the quark condensate vanishes for zero quark mass and the symmetry is not broken.

We also studied the fluctuations around the vacuum embeddings. In the massless quark limit there appear $(8-q-p+s)$ massless scalar bosons, which are identified with the NG bosons associated with the spontaneous symmetry breaking. For massive quarks the symmetry is explicitly broken by a quark mass and there appear massive pseudo-NG bosons. We showed that the pseudo-NG bosons satisfy the GMOR relation for a small quark mass by using the holographic description. We also obtained the effective action of the NG bosons at quartic order. This action is different from the one assumed in the Skyrme model.

At finite temperature we found that the rotational symmetry is broken by the vacuum configuration as in the zero temperature case. This corresponds to the chiral symmetry breaking in $\mathrm{QCD}_{s+1}$. We also found that there is a non-zero quark condensate $\langle\bar{\psi} \psi\rangle$ except for $T \rightarrow \infty$. The quark condensate vanishes and the chiral symmetry is restored in the high temperature limit. It will be interesting to study the theory at finite chemical potential $\mu$ as well as at finite temperature $T$. Then we will be able to obtain the phase diagram in the $(\mu, T)$ space and discuss the chiral phase transition in the $\mathrm{D} q / \mathrm{D} p$ model. 


\section{Acknowledgements}

The work of N.H. is partly supported by the Sasakawa Scientific Research Grant from The Japan Science Society.

\section{References}

[1] J. Maldacena, The large $N$ limit of superconformal field theories and supergravity, Adv. Theor. Math. Phys. 2 (1998) 231 arXiv:hep-th/9711200.

[2] S.S. Gubser, I.R. Klebanov and A.M. Polyakov, Gauge theory correlators from non-critical string theory, Phys. Lett. B428 (1998) 105 arXiv:hep-th/9802109].

[3] E. Witten, Anti de Sitter space and holography, Adv. Theor. Math. Phys. 2 (1998) 253 [arXiv:hep-th/9802150].

[4] O. Aharony, S.S. Gubser, J. Maldacena, H. Ooguri and Y. Oz, Large $N$ field theories, string theory and gravity, Phys. Rept. 323 (2000) 183 arXiv:hep-th/9905111.

[5] A. Karch and E. Katz, Adding flavor to AdS/CFT, JHEP 0206 (2002) 043 arXiv:hep-th/0205236.

[6] T. Sakai and J. Sonnenschein, Probing flavored mesons of confining gauge theories by supergravity, JHEP 0309 (2003) 047 arXiv:hep-th/0305049

[7] J. Babington, J. Erdmenger, N.J. Evans, Z. Guralnik and I. Kirsch, Chiral symmetry breaking and pions in non-supersymmetric gauge/gravity duals, Phys. Rev. D69 (2004) 066007 [arXiv:hep-th/0306018].

[8] M. Kruczenski, D. Mateos, R.C. Myers and D.J. Winters, Towards a holographic dual of large- $N_{c}$ QCD, JHEP 0405 (2004) 041 [arXiv:hep-th/0311270].

[9] N.J. Evans and J.P. Shock, Chiral dynamics from AdS space, Phys. Rev. D70 (2004) 046002 arXiv:hep-th/0403279.

[10] K. Ghoroku and M. Yahiro, Chiral symmetry breaking driven by dilaton, Phys. Lett. B604 (2004) 235 arXiv:hep-th/0408040. 
[11] D. Bak and H. U. Yee, Separation of spontaneous chiral symmetry breaking and confinement via AdS/CFT correspondence, Phys. Rev. D71 (2005) 046003 arXiv:hep-th/0412170.

[12] M.J. Rodriguez and P. Talavera, A 1+1 field theory spectrum from M theory, arXiv:hep-th/0508058.

[13] T. Sakai and S. Sugimoto, Low energy hadron physics in holographic QCD, Prog. Theor. Phys. 113 (2005) 843 [arXiv:hep-th/0412141].

[14] T. Sakai and S. Sugimoto, More on a holographic dual of QCD, Prog. Theor. Phys. 114 (2006) 1083 arXiv:hep-th/0507073.

[15] E. Antonyan, J.A. Harvey, S. Jensen and D. Kutasov, NJL and QCD from string theory, arXiv:hep-th/0604017.

[16] Y.h. Gao, W.s. $\mathrm{Xu}$ and D.f. Zeng, NGN, $\mathrm{QCD}_{2}$ and chiral phase transition from string theory, JHEP 0608 (2006) 018 arXiv:hep-th/0605138.

[17] E. Antonyan, J.A. Harvey and D. Kutasov, The Gross-Neveu model from string theory, Nucl. Phys. B776 (2007) 93 arXiv:hep-th/0608149].

[18] A. Basu and A. Maharana, Generalized Gross-Neveu models and chiral symmetry breaking from string theory, Phys. Rev. D75 (2007) 065005 arXiv:hep-th/0610087.

[19] E. Antonyan, J.A. Harvey and D. Kutasov, Chiral symmetry breaking from intersecting D-branes, Nucl. Phys. B784 (2007) 1 arXiv:hep-th/0608177.

[20] D. Gepner and S.S. Pal, Chiral symmetry breaking and restoration from holography, arXiv:hep-th/0608229.

[21] R. Casero, E. Kiritsis and A. Paredes, Chiral symmetry breaking as open string tachyon condensation, arXiv:hep-th/0702155.

[22] K. Hashimoto, T. Hirayama and A. Miwa, Holographic QCD and pion mass, JHEP 0706 (2007) 020 arXiv:hep-th/0703024.

[23] N. Evans and E. Threlfall, Quark mass in the Sakai-Sugimoto model of chiral symmetry breaking, arXiv:0706.3285 [hep-th]. 
[24] O. Bergman, S. Seki and J. Sonnenschein, Quark mass and condensate in HQCD, arXiv:0708.2839 [hep-th].

[25] A. Dhar and P. Nag, Sakai-Sugimoto model, tachyon condensation and chiral symmetry breaking, arXiv:0708.3233 [hep-th].

[26] K. Ghoroku, T. Sakaguchi, N. Uekusa and M. Yahiro, Flavor quark at high temperature from a holographic model, Phys. Rev. D71 (2005) 106002 arXiv:hep-th/0502088.

[27] O. Aharony, J. Sonnenschein and S. Yankielowicz, A holographic model of deconfinement and chiral symmetry restoration, Annals Phys. 322 (2007) 1420 arXiv:hep-th/0604161].

[28] A. Parnachev and D.A. Sahakyan, Chiral phase transition from string theory, Phys. Rev. Lett. 97 (2006) 111601 arXiv:hep-th/0604173.

[29] D. Mateos, R.C. Myers and R.M. Thomson, Holographic phase transitions with fundamental matter, Phys. Rev. Lett. 97 (2006) 091601 arXiv:hep-th/0605046.

[30] K. Peeters, J. Sonnenschein and M. Zamaklar, Holographic melting and related properties of mesons in a quark gluon plasma, Phys. Rev. D74 (2006) 106008 arXiv:hep-th/0606195.

[31] D. Mateos, R.C. Myers and R.M. Thomson, Thermodynamics of the brane, JHEP 0705 (2007) 067 [arXiv:hep-th/0701132].

[32] K.Y. Kim, S.J. Sin and I. Zahed, Dense hadronic matter in holographic QCD, arXiv:hep-th/0608046.

[33] N. Horigome and Y. Tanii, Holographic chiral phase transition with chemical potential, JHEP 0701 (2007) 072 arXiv:hep-th/0608198.

[34] A. Parnachev and D.A. Sahakyan, Photoemission with chemical potential from QCD gravity dual, Nucl. Phys. B768 (2007) 177 arXiv:hep-th/0610247.

[35] S. Nakamura, Y. Seo, S.J. Sin and K.P. Yogendran, A new phase at finite quark density from AdS/CFT, arXiv:hep-th/0611021. 
[36] S. Kobayashi, D. Mateos, S. Matsuura, R.C. Myers and R.M. Thomson, Holographic phase transitions at finite baryon density, JHEP 0702 (2007) 016 arXiv:hep-th/0611099.

[37] D. Mateos, S. Matsuura, R.C. Myers and R.M. Thomson, Holographic phase transitions at finite chemical potential, arXiv:0709.1225 [hep-th].

[38] M. Gell-Mann, R.J. Oakes and B. Renner, Behavior of current divergences under SU(3) × SU(3), Phys. Rev. 175 (1968) 2195.

[39] J. Polchinski, String Theory. Vol. 2: Superstring Theory and Beyond (Cambridge Univ. Press, 1998)

[40] N. Itzhaki, J.M. Maldacena, J. Sonnenschein and S. Yankielowicz, Supergravity and the large $N$ limit of theories with sixteen supercharges, Phys. Rev. D58 (1998) 046004 arXiv:hep-th/9802042.

[41] S.R. Coleman, There are no Goldstone bosons in two-dimensions, Commun. Math. Phys. 31 (1973) 259.

[42] D.J. Gross and A. Neveu, Dynamical symmetry breaking in asymptotically free field theories, Phys. Rev. D10 (1974) 3235.

[43] T.H.R. Skyrme, A nonlinear field theory, Proc. Roy. Soc. Lond. A260 (1961) 127.

[44] I. Zahed and G.E. Brown, The Skyrme model, Phys. Rept. 142 (1986) 1.

[45] E. Witten, Anti de Sitter space, thermal phase transition, and confinement in gauge theories, Adv. Theor. Math. Phys. 2 (1998) 505 arXiv:hep-th/9803131. 